\title{
The Neighborhood Configuration Model: a Framework To Distinguish Topological Relationships between Complex Volumes
}

\author{
Tao Chen \& Markus Schneider* \\ Department of Computer and Information Science and Engineering \\ University of Florida \\ Gainesville, FL 32611, USA \\ \{tachen, mschneid\}@cise.ufl.edu
}

\begin{abstract}
Topological relationships between spatial objects are considered to be important for spatial databases. They lead to topological predicates, which can be embedded into spatial queries as join or selection conditions. Before rushing into the implementation of topological predicates, topological relationships between spatial objects must be first understood and clarified. This requires a detailed study of a vast number of possible spatial configurations at the abstract level, and as a result, methods that are able to classify and identify as many as possible different spatial configurations are needed. While a lot of research has already been carried out for topological relationships in the $2 \mathrm{D}$ space, the investigation in the $3 \mathrm{D}$ space is rather neglected. Developed modeling strategies are mostly extensions from the popular 9-intersection model which has been originally designed for simple $2 \mathrm{D}$ spatial objects. We observe that a large number of topological relationships, especially the ones between two complex 3D objects are still not distinguished in these models. Thus, we propose a new modeling strategy that is based on point set topology. We explore all possible neighborhood configurations of an arbitrary point in the Euclidean space where two volume objects are embedded, and define corresponding neighborhood configuration flags. Then, by composing the Boolean values of all flags, we uniquely identify a topological relationship between two complex volume objects.
\end{abstract}

\section{Introduction}

Topological relationships like overlap, inside, or meet describe purely qualitative properties that characterize the relative positions of spatial objects and are preserved under affine transformations such as translation, scaling, and rotation. The exploration of topological relationships between spatial objects is an important topic in fields like artificial intelligence, cognitive science, geographical information systems (GIS), linguistics, psychology, robotics, spatial database

\footnotetext{
* This work was partially supported by the National Science Foundation under grant number NSF-IIS-0915914.
} 
systems, and qualitative spatial reasoning. From a database and GIS perspective, their development has been motivated by the need of formally defined topological predicates as filter conditions for spatial selections and spatial joins in spatial query languages and as a support for spatial data retrieval and analysis tasks.

The central conceptual approach, upon which almost all publications in this field have been based, is the 9-intersection model (9IM) [1]. This model checks the nine intersections of the boundary, interior, and exterior of a spatial object with the respective components of another spatial object for the topologically invariant criterion of non-emptiness. Extensions have been proposed to obtain more fine-grained topological predicates. However, the main focus has been on spatial objects in the 2D space; the study of topological relationships between spatial objects in the 3D space has been rare. An available strategy is to apply 9IM based models and to investigate the total number of relationships that can occur in reality between spatial objects in the 3D space. However, the third dimension introduces more complicated topological situations between 3D spatial objects. When directly applying the 9IM based models to 3D complex spatial objects like volumes, they suffer from a major problem, which we call the high granularity problem. That is, the 9IM considers the interior, exterior, and boundary point sets as the basic elements for empty and non-empty intersection tests, which ignores the fact that the interior, exterior, and the boundary of a spatial object are also complex spatial object parts, and may have multiple components. Thus, the interaction between any pair of the basic elements from two spatial objects can be complex, and empty or non-empty intersection results may not be enough to describe such interactions. For example, the boundary of a volume object is a closed surface object, which may have multiple components. Thus, the interaction between the boundaries of two volume objects is equivalent to the interaction between two complex surface objects, which can touch at a point, meet at a face, cross each other, or have touch, meet, and cross interactions coexist on one or more components. Since 9IM based models do not have the capability to handle these interaction details for their basic elements, a large number of topological relationships between complex volumes are not distinguished.

In this paper, we propose a new framework based on point set theory and point set topology to model topological relationships between two complex volume objects. We overcome the problems raised on the 9IM by investigating the interactions between two volumes at a much lower granularity. Instead of checking the intersections of the interior, boundary, and exterior of two volumes, we investigate the interaction of the two volumes within the neighborhood of any point in the Euclidean space where the two volumes rest, which we call the neighborhood configuration. We explore all possible neighborhood configurations, and define corresponding Boolean neighborhood configuration flags. By evaluating and composing the values of the neighborhood configuration flags for a given scenario with two complex volumes, we can obtain a binary encoding of the topological relationship between the two volumes. We show that our model yields more and thus a more fine-grained characterization of topological relationships between two complex volumes compared to 9IM. 


$$
\left(\begin{array}{ccc}
A^{\circ} \cap B^{\circ} \neq \varnothing A^{\circ} \cap \partial B \neq \varnothing A^{\circ} \cap B^{-} \neq \varnothing \\
\partial A \cap B^{\circ} \neq \varnothing \partial A \cap \partial B \neq \varnothing \partial A \cap B^{-} \neq \varnothing \\
A^{-} \cap B^{\circ} \neq \varnothing A^{-} \cap \partial B \neq \varnothing A^{-} \cap B^{-} \neq \varnothing
\end{array}\right)
$$

Fig. 1. The 9-intersection matrix for topological relationships.

Section 2 discusses related work about topological relationships. We propose our neighborhood configuration model (NCM) in Section 3, where we introduce in detail the definition of neighborhood configurations, the exploration of all possible neighborhood configurations, and the encoding of a topological relationship between two complex volumes. In Section 4, we compare our approach to the 9IM based models. Finally, Section 5 draws some conclusions.

\section{Related Work}

A special emphasis of spatial research has been put on the exploration of topological relationships (for example, overlap, inside, disjoint, meet) between spatial objects. An important approach for characterizing them rests on the so-called 9-intersection model, which employs point set theory and point set topology [1]. The model is based on the nine possible intersections of the boundary $(\partial A)$, interior $\left(A^{\circ}\right)$, and exterior $\left(A^{-}\right)$of a spatial object $A$ with the corresponding components of another object $B$. Each intersection is tested with regard to the topologically invariant criteria of emptiness and non-emptiness. The topological relationship between two spatial objects $A$ and $B$ can be expressed by evaluating the matrix in Figure 1. A total of $2^{9}=512$ different configurations are possible from which only a certain subset makes sense depending on the combination of spatial data types just considered. Several extensions based on the 9IM exist. Examples are the dimensionality extensions in $[2,3]$, the Voronoi-based extensions in [4], and the extensions to complex spatial objects in [5].

A topic that has been partially formally explored at the abstract level deals with topological relationships between simple 3D spatial objects. In [6], the author applies the 9-intersection model to simply-connected 3D spatial objects, that is, simple 3D lines, simple surfaces (no holes), and simple volumes (no cavities), in order to determine their topological relationships. A total of 8 topological relationships are distinguished between two simple volumes. Zlatanova has also investigated the possible 3D topological relationships in [7] by developing a complete set of negative conditions. The 9-intersection model for 3D can be extended with the dimensionality being considered. In [8], the values of the matrix elements are extended. Besides the $\varnothing$ and $\neg \varnothing$ symbols, the $*$ is used to indicate the omitted specification for the resulting set at this position, and the numbers $(0,1,2,3)$ refer to the dimensionality of the resulting set. Therefore, unlike the 1:1 mapping between the matrix with the topological relationships in the 9-intersection model, a matrix that contains a $*$ value represents a class of 
topological relationships. As a result, the topological relationships are clustered and manageable to the user. However, these 9IM based models suffer from the aforementioned high granularity problem due to the use of interior, exterior and boundary sets as basic elements.

The above models in common apply set theory to identify topological relationships. Thus, they can be categorized as point-set based topological relationships models. There are also other approaches that do not employ the point-set theory. The approach in [9] investigates topological predicates between cell complexes, which are structures from algebraic topology. It turns out that, due to limitations of cell complexes, the topological relationships between them are only a subset of the derived point-set based topological relationships. The topological relationships between 3D spatial objects that consist of a series of cell complexes can be described by the combination of relationships between those cells [10]. The Dimensional Model (DM) [11] is a model that is independent of the 9intersection model. It defines dimensional elements on a spatial object, and all the dimensional elements contribute to the final result. Three levels of details for the topological relationships are developed for different application purposes. The dimension model can distinguish some cases, especially meet cases that the 9-intersection model cannot identify. However, since it leaves the abstract topological space where only point sets are used, it is not clear how the dimensional elements can be constructed.

\section{The Neighborhood Configuration Model (NCM)}

In the following sections, we introduce in detail our new modeling strategy for topological relationships between 3D complex volumes. Section 3.1 gives an overview of our modeling strategy. We explore all possible neighborhood configurations for a given scenario of two spatial volume objects in Section 3.2. Finally, in Section 3.3, we show how the topological relationships can be identified and encoded with the neighborhood configuration flags.

\subsection{Overview}

In this paper, we are interested in complex volumes that may contain cavities or multiple components. A formal definition of complex volume objects can be found in [12], which models volumes as special infinite point sets in the threedimensional Euclidean space. Our approach is also based on point set theory and point set topology. The basic idea is to evaluate the values of a set of Boolean neighborhood configuration flags to determine the topological relationships between two volumes. Each neighborhood configuration flag indicates the existence or non-existence of a characteristic neighborhood configuration of the points in a given scenario. The neighborhood configuration of a point describes the ownerships of the points that are "near" the reference point. If the existence of a neighborhood configuration is detected, then the corresponding neighborhood configuration flag is set to true. For example, for a scenario that involves two 
volumes $A$ and $B$, if there exists a point $p$ whose neighboring points all belong to both $A$ and $B$, then the corresponding neighborhood configuration flag exist_nei_in_overlap (see Definition 1(1)) is set to true. Later, this neighborhood configuration flag contributes to the determination of the topological relationships between $A$ and $B$.

\subsection{Exploring Neighborhood Configuration Information}

In this section, we explore all neighborhood configurations. We begin with some needed topological and metric concepts. Let $\mathbb{R}$ be the set of real numbers, $\mathbb{R}^{+}=$ $\{x \in \mathbb{R} \mid x>0\}$, and $\mathbb{R}^{3}$ be the three-dimensional Euclidean space. We assume the existence of a Euclidean distance function $d: \mathbb{R}^{3} \times \mathbb{R}^{3} \rightarrow \mathbb{R}$ with $d(p, q)=$ $d\left(\left(x_{1}, y_{1}, z_{1}\right),\left(x_{2}, y_{2}, z_{2}\right)\right)=\sqrt{\left(x_{1}-x_{2}\right)^{2}+\left(y_{1}-y_{2}\right)^{2}+\left(z_{1}-z_{2}\right)^{2}}$. Then for any point $p \in \mathbb{R}^{3}$ and $r \in \mathbb{R}^{+}$, the set $N_{r}(p)=\left\{q \in \mathbb{R}^{3} \mid d(p, q) \leq r\right\}$ is called the closed ball with radius $r$ and center $p$. Let $\epsilon$ denote a small positive value $(\epsilon \rightarrow 0)$; we call the closed ball $N_{\epsilon}(p)$ the (closed) neighborhood of point $p$. We can think of the neighborhood around $p$ as a tiny closed ball containing all points in $\mathbb{R}^{3}$ that are "nearest" to $p$.

With the necessary topological concepts introduced, we now explore the possible neighborhood configurations of points in a scenario that involves two volume objects. Let us assume two volumes $A$ and $B$ that are point sets in $\mathbb{R}^{3}$ $\left(A \subset \mathbb{R}^{3}, B \subset \mathbb{R}^{3}\right)$. Then given a point $p \in \mathbb{R}^{3}$, any point $q$ in its neighborhood $N_{\epsilon}(p)\left(q \in N_{\epsilon}(p)\right)$ has one of the following four possible ownerships: (i) $q \in A \wedge q \notin B$, (ii) $q \notin A \wedge q \in B$, (iii) $q \in A \wedge q \in B$, and (iv) $q \notin A \wedge q \notin B$. As a result, we can describe the ownership of a neighborhood by combining the ownerships of all points in it. We call this ownership description of a neighborhood the neighborhood configuration. With the four possible ownerships of a single point, we can obtain a total of $2^{4}-1=15$ possible ownership combinations for a neighborhood (the case where none of the four ownerships exists in a neighborhood is not possible). In other words, 15 neighborhood configurations are possible for any point in $\mathbb{R}^{3}$ where $A$ and $B$ are embedded. As a result, based on Definition 1 we can define 15 corresponding neighborhood configuration flags for a scenario that involves $A$ and $B$.

Definition 1. Let $A, B \subset \mathbb{R}^{3}, p \in \mathbb{R}^{3}$, and $N_{\epsilon}(p)$ be a neighborhood of $p$ with a tiny radius $\epsilon$. We first define four ownership flags for $p$ :

$$
\begin{aligned}
& \alpha(A, B, p) \Leftrightarrow \exists x \in N_{\epsilon}(p): x \in A \wedge x \in B \\
& \beta(A, B, p) \Leftrightarrow \exists x \in N_{\epsilon}(p): x \in A \wedge x \notin B \\
& \gamma(A, B, p) \Leftrightarrow \exists x \in N_{\epsilon}(p): x \notin A \wedge x \in B \\
& \lambda(A, B, p) \Leftrightarrow \exists x \in N_{\epsilon}(p): x \notin A \wedge x \notin B
\end{aligned}
$$

Then we define the following 15 neighborhood configuration flags for a scenario involves two objects $A$ and $B$ :

(1) exist_nei_in_overlap $(A, B)$

$$
\Leftrightarrow \exists p \in \mathbb{R}^{3}: \alpha(A, B, p) \wedge \neg \beta(A, B, p) \wedge \neg \gamma(A, B, p) \wedge \neg \lambda(A, B, p)
$$

(2) exist_nei_in_op1 $(A, B)$

$$
\Leftrightarrow \exists p \in \mathbb{R}^{3}: \neg \alpha(A, B, p) \wedge \beta(A, B, p) \wedge \neg \gamma(A, B, p) \wedge \neg \lambda(A, B, p)
$$




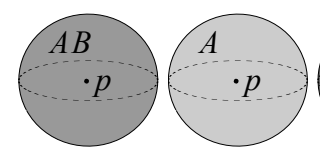

(1)

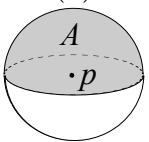

(9)

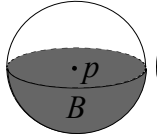

(10)

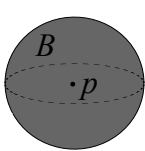

(3)

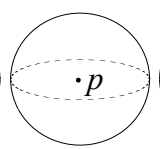

(4)

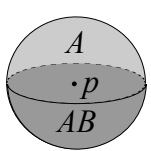

(5)

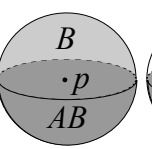

(6)

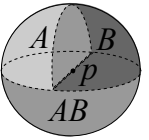

(14)

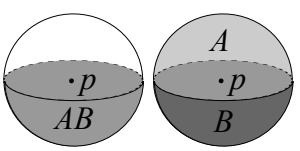

(7)

(8)

(11)

$(12)$

(13)

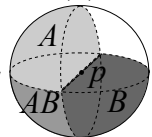

(15)

Fig. 2. The drawing of the 15 neighborhood configurations for point $p$.

(3) exist_nei_in_op2 $(A, B)$

$\Leftrightarrow \exists p \in \mathbb{R}^{3}: \neg \alpha(A, B, p) \wedge \neg \beta(A, B, p) \wedge \gamma(A, B, p) \wedge \neg \lambda(A, B, p)$

(4) exist_nei_in_ext $(A, B)$

$\Leftrightarrow \exists p \in \mathbb{R}^{3}: \neg \alpha(A, B, p) \wedge \neg \beta(A, B, p) \wedge \neg \gamma(A, B, p) \wedge \lambda(A, B, p)$

(5) exist_nei_contain_overlap_op $1(A, B)$

$\Leftrightarrow \exists p \in \mathbb{R}^{3}: \alpha(A, B, p) \wedge \beta(A, B, p) \wedge \neg \gamma(A, B, p) \wedge \neg \lambda(A, B, p)$

(6) exist_nei_contain_overlap_op2 $(A, B)$

$\Leftrightarrow \exists p \in \mathbb{R}^{3}: \alpha(A, B, p) \wedge \neg \beta(A, B, p) \wedge \gamma(A, B, p) \wedge \neg \lambda(A, B, p)$

(7) exist_nei_contain_overlap_ext $(A, B)$

$\Leftrightarrow \exists p \in \mathbb{R}^{3}: \alpha(A, B, p) \wedge \neg \beta(A, B, p) \wedge \neg \gamma(A, B, p) \wedge \lambda(A, B, p)$

(8) exist_nei_contain_op1_op2 $(A, B)$ $\Leftrightarrow \exists p \in \mathbb{R}^{3}: \neg \alpha(A, B, p) \wedge \beta(A, B, p) \wedge \gamma(A, B, p) \wedge \neg \lambda(A, B, p)$

(9) exist_nei_contain_op1_ext $(A, B)$ $\Leftrightarrow \exists p \in \mathbb{R}^{3}: \neg \alpha(A, B, p) \wedge \beta(A, B, p) \wedge \neg \gamma(A, B, p) \wedge \lambda(A, B, p)$

(10) exist_nei_contain_op2_ext $(A, B)$ $\Leftrightarrow \exists p \in \mathbb{R}^{3}: \neg \alpha(A, B, p) \wedge \neg \beta(A, B, p) \wedge \gamma(A, B, p) \wedge \lambda(A, B, p)$

(11) exist_nei_contain_op1_op2_ext $(A, B)$ $\Leftrightarrow \exists p \in \mathbb{R}^{3}: \neg \alpha(A, B, p) \wedge \beta(A, B, p) \wedge \gamma(A, B, p) \wedge \lambda(A, B, p)$

(12) exist_nei_contain_op2_overlap_ext $(A, B)$ $\Leftrightarrow \exists p \in \mathbb{R}^{3}: \alpha(A, B, p) \wedge \neg \beta(A, B, p) \wedge \gamma(A, B, p) \wedge \lambda(A, B, p)$

(13) exist_nei_contain_op1_overlap_ext $(A, B)$ $\Leftrightarrow \exists p \in \mathbb{R}^{3}: \alpha(A, B, p) \wedge \beta(A, B, p) \wedge \neg \gamma(A, B, p) \wedge \lambda(A, B, p)$

(14) exist_nei_contain_op1_op2_overlap $(A, B)$ $\Leftrightarrow \exists p \in \mathbb{R}^{3}: \alpha(A, B, p) \wedge \beta(A, B, p) \wedge \gamma(A, B, p) \wedge \neg \lambda(A, B, p)$

(15) exist_nei_contain_op1_op2_overlap_ext $(A, B)$ $\Leftrightarrow \exists p \in \mathbb{R}^{3}: \alpha(A, B, p) \wedge \beta(A, B, p) \wedge \gamma(A, B, p) \wedge \lambda(A, B, p)$

The above 15 neighborhood configuration flags identify all possible interactions between two spatial volumes $A$ and $B$ in $\mathbb{R}^{3}$ at any single point. We demonstrate the validity of these neighborhood configuration flags by creating drawings for the corresponding neighborhood configurations in Figure 2. For example, if flag (8) yields true (Figure 2(8)), then it means that there exists a point whose neighborhood consists of points that are only from the first operand object $A$ and points that are only from the second operand object $B$. The value 


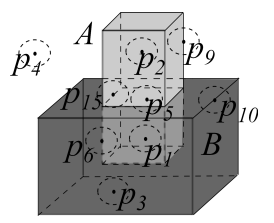

(111111001100001)

(a)

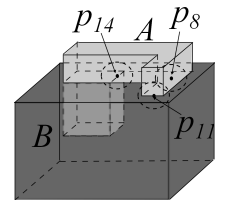

$(111111011110011)$

(b)

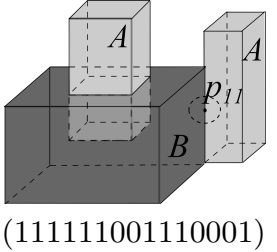

(c)

Fig. 3. Examples of topological relationship scenarios and their corresponding topological relationship encodings.

true of this flag (exist_nei_contain_op1_op2) further indicates the existence of a meeting on face topological relationship between two volume objects $A$ and $B$.

\subsection{Topological Relationship Encoding with Neighborhood Configuration Flags}

In the previous section, we have introduced 15 neighborhood configuration flags for two complex volume objects embedded in the Euclidean space $\mathbb{R}^{3}$. These flags capture all topological situations for two volumes at all points in the Euclidean space $\mathbb{R}^{3}$. In other words, we have identified the topological relationships between two volumes at a very low granularity level, which involves a small neighborhood of a point. Thus, to determine the topological relationships between two volumes, we just need to collect the values of all 15 neighborhood configuration flags. First, let $F$ denote an array that stores the values of all 15 neighborhood configuration flags that are defined in the previous section. Further, we assume the ordering of the flags stored in $F$ is the same as in Definition 1. Definition 2 defines the topological relationship encoding for two volume objects.

Definition 2. Let $A, B$ be two volume objects in $\mathbb{R}^{3}\left(A, B \subset \mathbb{R}^{3}\right)$, and let $F V$ denote the function that encodes the topological interaction between two volumes with respect to the ith neighborhood configuration flag's value $(1 \leq i \leq 15)$. Then we have:

$$
F V(A, B, i)=\left\{\begin{array}{lll}
0 & \text { if } & F[i] \text { yields false for } A \text { and } B \\
1 & \text { if } & F[i] \text { yields true for } A \text { and } B
\end{array}\right.
$$

Thus, we can use a 15 bit binary array to encode the topological relationship between $A$ and $B$. The definition for the topological relationship encoding TRE is given as:

$$
\operatorname{TRE}(A, B)=(F V(A, B, 0) F V(A, B, 1) \ldots F V(A, B, 14))
$$

Definition 2 introduces a 15 bits binary representation for the topological relationships between two volume objects. Figure 3 presents three examples of the topological relationship encodings. The encoding in Figure $3 \mathrm{a}$ indicates that 9 topological relationship flags yield true for the scenario involving $A$ and $B$, which are exist_nei_in_overlap, exist_nei_in_op1, 
exist_nei_in_op2, exist_nei_in_ext, exist_nei_contain_overlap_op1, exist_nei_contain_overlap_op2, exist_nei_contain_op1_ext, exist_nei_contain_op2_ext, and exist_nei_contain_op1_op2_overlap_ext. To demonstrate the validity of the encoding, we have marked the corresponding points $p_{i}$ that validates the true value of flag $F[i]$. In Figure $3 \mathrm{~b}$, three additional flags, which are exist_nei_contain_op1_op2, exist_nei_contain_op1_op2_ext, and exist_nei_contain_op1_op2_overlap, become true due to the points $p_{8}, p_{11}$, and $p_{14}$ respectively. Therefore, Figure 3 presents three different topological relationships that can be distinguished by our encoding, which are overlap encoded by 111111001100001, overlap with meet on face encoded by 111111011110011, and overlap with meet on edge encoded by 111111001110001 .

As a result, we obtain a total of $2^{15}=32768$ possible topological relationship encoding values, which implies a total of 32768 possible topological relationships between two volume objects. However, not all encoding values represent valid topological relationships. We call a topological relationship encoding valid for two volumes if, and only if, it can be derived from a real world scenario that involves two volume objects. For example, there does not exist a real world scenario with the topological relationship encoding 000000000000000. Thus, the open questions are now (i) which are the valid topological relationship encodings for two complex volumes, (ii) which are the valid topological relationship encodings for two simple volumes, and (iii) what their numbers are. However, due to space limitation, our goal is only to propose the basic modeling strategy in this paper; thus, we leave these questions open.

\section{The Comparison of NCM with 9IM based Models}

Our neighborhood configuration model and the 9-intersection based models share an important basis, which is point set theory and point set topology. This basis is important because it enables the modeling strategies to stay in the abstract topological space where only point sets are used and the discrete representation of a spatial object is not relevant. As a result, models based on point sets are more general than other models that leave the abstract topological space. No matter what data representation of a spatial object is used, the models based on the point sets will always work. On the other hand, models based on one particular representation of spatial objects may not work or may yields different results on another representation of the same spatial objects. For example, the dimensional model [11] (DM) is based on the dimensional elements of spatial objects, which determines the corner points of a region object as $0 \mathrm{D}$ elements. It highly depends on the representation of a spatial object, and it yields different results for a circle with a smooth boundary and a circle with its boundary approximated with a set of connected segments. Therefore, in this section, we only compare our model with the 9IM model, and show that we can distinguish more topological relationships between two volume objects.

According to [6], 8 topological relationships are distinguished between two simple $3 \mathrm{D}$ volume objects. Figure 4 shows the 8 configurations and their cor- 


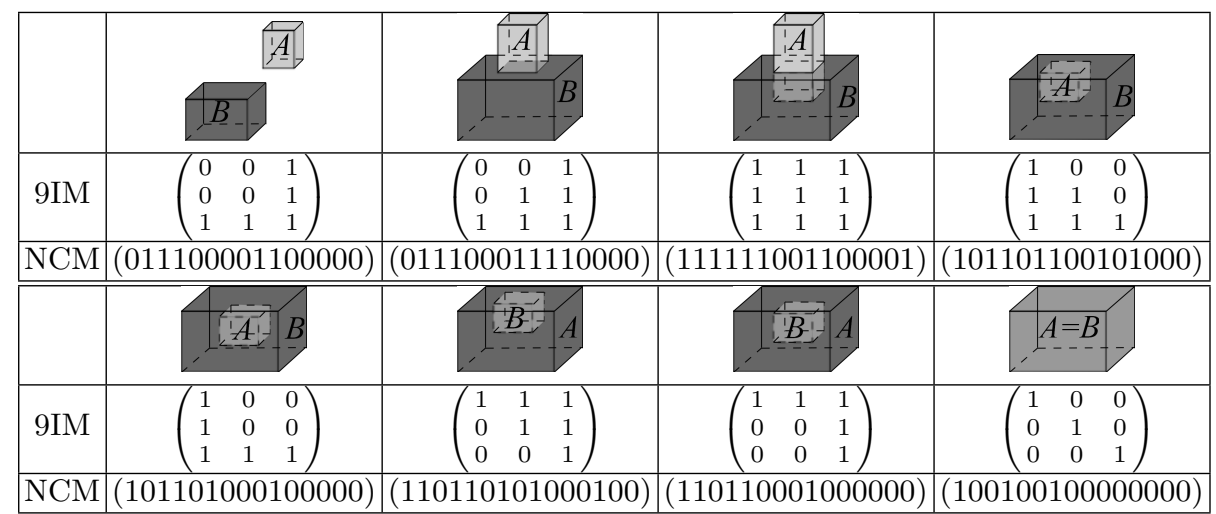

Fig. 4. The 8 topological relationships between two volumes that can be distinguished with both 9IM and NCM.

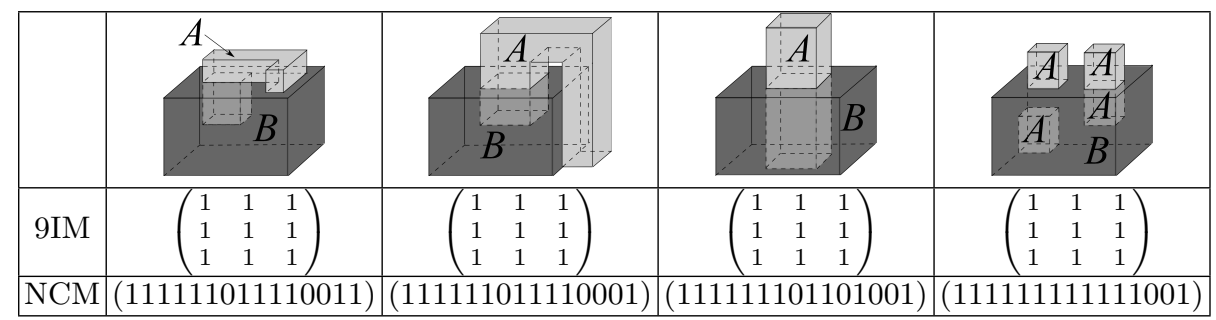

Fig. 5. The 4 topological relationships that can be distinguished with NCM but not 9IM.

responding 9IM matrices. To demonstrate that our model can also distinguish these 8 topological relationships, we also list our topological relationship encoding for each configuration in Figure 4. We observe that for all 8 topological relationships the topological relationship encodings are also different.

Moreover, the 9IM model only picks up the dominating topological relationships such as overlap, and ignores other topological relationship factors that may also exist at the same time, e.g. meet at a face. However, since we start with a very small neighborhood of each point, we are able to capture more fine-grained topological relationships. In Figure 5, we have listed 4 topological relationships that can be distinguished with our NCM model but are all identified simply as overlap in the 9IM model. Apart from the overlap relationship between $A$ and $B$, the topological relationships like $A$ meets $B$ on a face from the outside (first and second scenario in Figure 5), $A$ meets $B$ on a face from the inside of $B$ (third scenario in Figure 5), and $A$ has a component inside $B$ and touches $B$ on a face (fourth scenario in Figure 5) are all distinguished in our model.

Therefore, we can conclude that our NCM model is more powerful than 9IM based models in terms of differentiating topological relationships between two volume objects, especially complex volumes. 


\section{Conclusion and Future Work}

In this paper, we have introduced a novel framework for distinguishing topological relationships between two volume objects. Our approach is based on point set theory and point set topology, and by identifying the neighborhood configurations of all points, we can encode and distinguish the topological relationships. We have also made a comparison between our model and the popular 9IM model in an informal manner, and have shown that we can distinguish more topological relationships than the 9IM can. However, we were not able to explore all topological relationships that our model can distinguish in a systematical way due to space limitation. Thus, in the future, our goal is to find the answer to the open questions that we mentioned at the end of Section 3.3, and further extend our model to other complex 3D spatial objects like surfaces and lines.

\section{References}

1. Egenhofer, M., Herring, J.: A Mathematical Framework for the Definition of Topological Relationships. In: Int. Symp. on Spatial Data Handling. (1990) 803-813

2. Clementini, E., Felice, P.D., Oosterom, P.: A Small Set of Formal Topological Relationships Suitable for End-user Interaction. In: 3rd Int. Symp. on Advances in Spatial Databases. (1993) 277-295

3. McKenney, M., Pauly, A., Praing, R., Schneider, M.: Dimension-refined Topological Predicates. In: 13th ACM Symp. on Geographic Information Systems (ACM GIS). (2005) 240-249

4. Chen, J., Li, C., Li, Z., Gold, C.: A Voronoi-based 9-intersection Model for Spatial Relations. International Journal of Geographical Information Science 15(3) (2001) 201-220

5. Schneider, M., Behr, T.: Topological Relationships between Complex Spatial Objects. ACM Trans. on Database Systems (TODS) 31(1) (2006) 39-81

6. Egenhofer, M.J.: Topological Relations in 3D. Technical report (1995)

7. Zlatanova, S.: On 3D Topological Relationships. In: 11th Int. Conf. on Database and Expert Systems Applications (DEXA). (2000) 913

8. Borrmann, A., van Treeck, C., Rank, E.: Towards a 3D Spatial Query Language for Building Information Models. In: Proceedings of the Joint International Conference for Computing and Decision Making in Civil and Building Engineering. (2006)

9. Pigot, S.: Topological Models for 3D Spatial Information Systems. In: International Conference on Computer Assisted Cartography(Auto-Carto). (1991) 368-392

10. Guo, W., Zhan, P., Chen, J.: Topological Data Modeling for 3D GIS. Int. Archives of Photogrammetry and Remote Sensing 32(4) (1998) 657-661

11. Billen, R., Zlatanova, S., Mathonet, P., Boniver, F.: The Dimensional Model: a Framework To Distinguish Spatial Relationships. In: Int. Symp. on Advances in Spatial Databases. (2002) 285-298

12. Schneider, M., Weinrich, B.E.: An Abstract Model of Three-Dimensional Spatial Data Types. In: 12th ACM Symp. on Geographic Information Systems (ACM GIS). (2004) 67-72 